October 15, 2015

\title{
High Energy Scatterings in Higher Dimensional Theories
}

\author{
Jnanadeva Maharana ${ }^{1}$ \\ E-mail maharana@iopb.res.in \\ Institute of Physics \\ Bhubaneswar - 751005, India \\ and \\ Max-Planck-Institut für Gravitationsphysik (Albert-Einstein-Institut), \\ DE-14476 Potsdam, Germany
}

\begin{abstract}
The high energy behavior of scattering amplitudes in spacetime dimensions, $D>4$, is investigated. The bound on total cross sections, $\sigma_{t} \leq$ Constant $(\log s)^{D-2}$, $D \geq 4$ has been obtained in the past under usual assumptions. I derive new bounds on scattering amplitudes in the region $|t|<T_{0}, t$ being momentum transfer squared and $T_{0}$ is a constant. The existence of a zero-free region for the amplitude, in complex t-plane, is proved. I prove stronger upper and lower bounds for the absorptive amplitude in the domain $0<t<T_{0}$.
\end{abstract}

\footnotetext{
${ }^{1}$ In memory of my Sumitra
} 
The Froissart-Martin bound restricts the growth of hadronic total cross sections, $\sigma_{t}(s)$, at high energies. Heisenberg [1], in 1952, argued that total cross sections may grow as fast as $(\ln s)^{2}$ at high energies. The arguments were based on his intuitions and he supported them by deriving the energy dependence of $\sigma_{t}$ from the Born-Infeld action for a scalar field.

Let us summarize the essential ingredients for derivation of rigorous results in strong interactions. These results are especially important in the context of hadronic reactions at asymptotic energies. We refer to books and reviews [2, 4, 5] for detail discussions and references. We consider scattering of scalar particles though out. The derivation of exact results rest on four pillars based on axiomatic field theory: (A1) Unitarity of S-matrix.

(A2) The amplitude, for fixed $s$, is analytic in complex $\cos \theta$-plane inside the LehmannMartin ellipse; $s$ and $\theta$ being the energy squared and scattering angles, respectively, in the center of mass frame. The foci of the ellipse lie at $(-1,+1)$ and its semi-major axis is $\cos \theta_{0}=1+2 t_{0} / s, t_{0}$ being an $s$-independent constant. For many hadronic processes, $t_{0}=4 m_{\pi}^{2}$. The partial wave expansion of the amplitude, $F(s, t)$, converges absolutely inside Lehmann-Martin ellipse,

$$
F(s, t)=\frac{\sqrt{s}}{k} \sum_{l=0}^{\infty}(2 l+1) f_{l}(s) P_{l}(\cos \theta)
$$

$P_{l}(\cos \theta)$ is analytic in $\cos \theta$, so is $F(s, t)$ in $t, \cos \theta=1+2 t / s ; k$ being the c.m. momentum. Unitarity bounds on the partial-wave amplitudes are

$$
0 \leq\left|f_{l}(s)\right|^{2} \leq \operatorname{Im} f_{l}(s) \leq 1
$$

(A3). Polynomial boundedness inside Lehmann-Martin ellipse [6]. For $0 \leq t \leq t_{0}$

$$
|F(s, t)|<_{|s| \rightarrow \infty}|s|^{N}
$$

$\mathrm{N}$ being a positive integer.

(A4). $F(s, t)$ is analytic in the complex $s$-plane. There are cuts in the s-plane as a consequence of $s$-channel unitarity and crossing symmetry - crossing is a requirement. The Froissart-Martin bound is $[7,8]$

$$
\sigma_{t}(s)<\frac{4 \pi}{t_{0}-\epsilon}\left[\ln \left(s / s_{0}\right)\right]^{2}
$$

$\epsilon$ is an arbitrary small constant and $s_{0}$ is an undetermined energy scale. However, recently it has been shown [9] that $s_{0}{ }^{-1}=17 \pi \sqrt{\pi / 2} m_{\pi}{ }^{-2}$ for $\pi \pi$ scattering. There are host of important, exact and well tested bounds on $[2,4,5]$

$$
\sigma_{t}, \sigma_{e l},\left(\frac{d \sigma}{d t}\right)_{e l}, b(s), \mathbf{h}_{d}=\left.\frac{d \sigma}{d t}\right|_{t=0}
$$


where $b(s)$ is the slope of the elastic diffraction peak and $\mathbf{h}_{b}$ is its height at $t=0$. The purpose of this article is to derive some important bounds for amplitudes describing scattering in $D>4$. The bounds on $\sigma_{t}$ and slope of the diffraction peak associated with the imaginary part of the amplitude etc. have been derived in the past $[10,11]$. I derive new bounds on the elastic amplitude $F^{\lambda}(s, t)$, defined below, in a region $|t| \leq T_{0}, T_{0}$ being a constant. This result extends the previously obtained results of $[10,11]$ to a region alluded to above. Moreover, a bound is derived on the distribution of zeros of $F^{\lambda}(s, t)$ in the complex $t$-plane. The radius of the zero-free circle shrinks as $(1 / \ln s)^{2}$. I prove that the absorptive amplitude is bounded from above and below in the domain $0<t<T_{0}$. There is a close relationship between the energy dependence of $b(s)$ and the zeros of $F^{\lambda}(s, t)$. The assumptions leading to these bounds are stated in sequel; although these are quite general, they do not follow from axiomatic field theory like (A1)-(A4) for $D=4$ case.

We recall that, for $D>4$, the elastic scattering amplitude of spinless particles admits partial wave expansion as demonstrated by Soldate [12]. The amplitude depends on $s$ and $\cos \theta$. The ultraspherical Jacobi function, the Gegenbauer polynomials [13, 14], are the basis as these are the 'spherical harmonics' associated with the $S O(D-1)$ rotation group. The amplitude, $F^{\lambda}(s, t)$, is expanded as

$$
F^{\lambda}(s, t)=A_{1} s^{-\lambda+1 / 2} \sum_{l=0}^{\infty}(l+\lambda) f_{l}^{\lambda}(s) C_{l}^{\lambda}(t)(1+2 t / s)
$$

where $\lambda=\frac{1}{2}(D-3)$ and $(s, t)$ are the usual Mandelstam variables. $A_{1}=2^{4 \lambda+3} \pi^{\lambda} \Gamma(\lambda)$, independent of $s$ and $t$. $C_{l}^{\lambda}(x)$ are Gegenbauer polynomials satisfying orthogonality conditions with weight factor $\left(1-x^{2}\right)^{\frac{D}{2}-1},-1 \leq x \leq+1$ [13]. The expansion (6) converges in the domain $-1 \leq \cos \theta \leq+1$ [13]. The partial-wave amplitudes, $\left\{f_{l}^{\lambda}(s)\right\}$, satisfy the unitarity constraints:

$$
0 \leq\left|f_{l}^{\lambda}(s)\right|^{2} \leq \operatorname{Im} f_{l}^{\lambda}(s) \leq 1
$$

as derived in [12].

Additional assumptions are needed to derive analog of Froissart-Martin bound (4). The existence of extended domain of analyticity for (6) is not proven for the theories in $D>4$, unlike the $D=4$ case. Thus two reasonable requirements were imposed in $[10,11]$. These are: (I) The domain of convergence, $\mathbf{D}$, of $F^{\lambda}(s, t)$ is an extended ellipse with the semimajor axis $1+2 T_{0} / s$ in the $t$-plane. (II) The amplitude is polynomially bounded: $\left|F^{\lambda}(s, t)\right|<C s^{N}$ in $\mathbf{D}$; C and $\mathrm{N}$ are undetermined constants, $N \in \mathbf{R}$. The domain of convergence of Gegenbauer polynomial is $[-1,+1]$ and a theorem (see Theorem 9.1.1 of [13]) states the convergence properties of functions such as $F^{\lambda}(s, t)$. The partial wave amplitudes $f_{l}^{\lambda}(s)$ decay exponentially with $l[10,11]$ under the assumptions (I) and (II). Therefore, the expansion (6) can be truncated at $L=\frac{1}{2}(N-1) \sqrt{s / T_{0}}$ lns and importantly, $L$ is $D$-independent. Consequently, $\sigma_{t}$ is bounded $[10,11]$ as

$$
\sigma_{t} \leq C_{0}(\ln s)^{D-2}
$$


Note that $\ln s$ is to be understood as $\ln (s / \hat{s}), \hat{s}$ is like $s_{0}$ which scales $s$. Important bounds on $F^{\lambda}(s, t)$ and its absorptive part are derived by utilizing crucial properties of $C_{l}^{\lambda}(x)$ and the assumptions (I) and (II). Let us summarize the useful inequalities as $[13]$.

Lemma 1. For $1<x_{1}<x_{2}<1+T_{0} / s, C_{l}^{\lambda}\left(x_{1}\right)<C_{l}^{\lambda}\left(x_{2}\right)$.

Proof. Define $z=\frac{1}{2}(1-x)$. For $x>1, z<0$. $C_{l}^{\lambda}(x)$, as a hypergeometric function is expanded as [14]

$$
C_{l}^{\lambda}(x)=\frac{\Gamma\left(\frac{\lambda+1}{2}\right)}{\Gamma(2 \lambda)} \sum_{k=0}^{l} \frac{\Gamma(2 \lambda+n+k)}{k !(l-k) ! \Gamma(\lambda+k+1 / 2)}(-z)^{k}
$$

Notice: (i) Coefficients of $(-z)^{k}$ are positive. (ii) For $1<x_{1}<x_{2}$, each term in the expansion (9), $B_{k}\left(z_{1}\right)<B_{k}\left(z_{2}\right)$ and therefore, $C_{l}^{\lambda}\left(x_{1}\right)<C_{l}^{\lambda}\left(x_{2}\right)$ in the range of interest. Moreover, $C_{l}^{\lambda}(x)$ oscillates in the physical region, $-1 \leq x<+1, x=\cos \theta$, $t<0$. We derive following bound on the modulus of the amplitude, $\left|F^{\lambda}(s, t)\right|, 0<$ $|t|<T_{0}$, utilizing the Lemma 1 and noting that $C_{l}^{\lambda}(1+2 t / s) \leq C_{l}^{\lambda}(1+2|t| / s)$.

Theorem. The modulus $\left|F^{\lambda}(s, t)\right|$ is bounded as

$$
\left|F^{\lambda}\right| \leq A_{2}\left(\frac{1}{|t|}\right)^{\frac{\lambda+1}{2}}\left(\frac{1}{T_{0}}\right)^{\lambda / 2} s^{1+(N-1) \sqrt{\frac{|t|}{T_{0}}}}(\ln s)^{\lambda}
$$

$0<|t|<T_{0}$ and $A_{2}=2 \lambda A_{1} 2^{-2 \lambda-1} \Gamma^{-2}(\lambda+1)$.

Proof. The proof proceeds through the following steps.

Decompose $F^{\lambda}(s, t)$ as

$$
F^{\lambda}(s, t)=F^{(1) \lambda}(s, t)+F^{(2) \lambda}(s, t)
$$

The expansion of $F^{(1)}(s, t)$ is terminated at $l=L$ and expansion of $F^{(2)}$ is from $L+1$ to $\infty$. Therefore,

$$
F^{(1) \lambda}(s, t) \leq A_{1} s^{-\lambda+1 / 2} \sum_{l=0}^{L}(l+\lambda)\left|f_{l}^{\lambda}\right| C_{l}^{\lambda}\left(1+\frac{2|t|}{s}\right)
$$

Invoking unitarity and setting $\left|f_{l}^{\lambda}(s)\right|=1$; (12) reduces to a sum: $\sum(l+\lambda) C_{l}^{\lambda}(1+$ $2|t| / s)$. For large $s$,

$$
\sum_{l=0}^{L}(l+\lambda) C_{l}^{\lambda}(1+2|t| / s) \sim C_{1}\left(\sqrt{\frac{s}{|t|}}\right)^{\frac{1+\lambda}{2}} L^{\lambda} \exp \left(2 L \sqrt{\frac{s}{|t|}}\right)
$$

Note $L=\frac{1}{2}(N-1) \sqrt{\frac{s}{T_{0}}} \ln s$ and $C_{1}=2 \lambda 2^{-2(\lambda+1)} \Gamma(\lambda+1)$. Thus the desired bound is

$$
\begin{aligned}
\left|F^{(1) \lambda}(s, t)\right| \leq \quad & A_{2}\left(\frac{N-1}{2}\right)^{\lambda}\left(\frac{1}{|t|}\right)^{\frac{1+\lambda}{2}}\left(\frac{1}{T_{0}}\right)^{\lambda / 2} \\
& s(\ln s)^{\lambda} \exp \left(2 L \sqrt{\frac{|t|}{s}}\right)
\end{aligned}
$$


with $A_{2}=A_{1} C_{1}$. Let us turn the attention on $F^{(2) \lambda}$. We argue, as before, to convince the reader that

$$
\left|F^{(2) \lambda}(s, t)\right| \leq A_{1} s^{-\lambda+1 / 2} \sum_{L+1}^{\infty}(l+\lambda)\left|f_{l}^{\lambda}\right|(s) C_{l}^{\lambda}(1+2|t| / s)
$$

and re-express the right hand side (15) as

$$
\begin{aligned}
\left|F^{(2) \lambda}(s, t)\right| \leq \quad & A_{1} s^{-\lambda+1 / 2} \sum_{L=1}^{\infty}\left[\sqrt{(l+\lambda)} \frac{C_{l}^{\lambda}(1+2|t| / s)}{\sqrt{C_{l}^{\lambda}\left(1+2 T_{1} / s\right)}}\right] \\
& \times\left[\sqrt{(l+\lambda)} \sqrt{C_{l}^{\lambda}\left(1+2 T_{1} / s\right)}\left|f_{l}^{\lambda}(s)\right|\right]
\end{aligned}
$$

where $|t|<T_{1}=T_{0}-\delta_{1} ; \delta_{1}>0$ but infinitesimally small. Now, invoke the Schwarz inequality on the r.h.s of (16)

$$
F^{(2) \lambda}(s, t) \leq A_{1} s^{-\lambda+1 / 2} \mathcal{S}_{1} \mathcal{S}_{2}
$$

where

$$
\begin{aligned}
& \mathcal{S}_{1}=\left[\sum_{L+1}^{\infty}(l+\lambda) \frac{\left(C_{l}^{\lambda}(1+2|t| / s)\right)^{2}}{C_{l}^{\lambda}\left(1+2 T_{1} / s\right)}\right]^{1 / 2} \\
& \mathcal{S}_{2}=\left[\sum_{L+1}^{\infty}(l+\lambda)\left|f_{l}^{\lambda}\right|^{2} C_{l}^{\lambda}\left(1+2 T_{1} / s\right)\right]^{1 / 2}
\end{aligned}
$$

In what follows, I outline the estimation of the upper bounds to $F^{(2) \lambda}(s, t)$ which is a generalization of the proof for the case $\lambda=1 / 2$. The term $\mathcal{S}_{1}$ involves ratio of two Gegenbauer polynomials with arguments $(1+2|t| / s)$ and $\left(1+2 T_{1} / s\right)$. In the large $s$, limit $\mathcal{S}_{1} \sim(\ln s)^{\lambda-1}$. For $\mathcal{S}_{2}$, invoke unitarity, $\left|f_{l}^{\lambda}\right|^{2} \leq 1$, and polynomial boundedness, (recall $\left|F^{(2) \lambda}(s, t)\right|<C s^{N}$ ), to give suppressed the energy dependence with appropriate choice of $\mathrm{C}$ (see [5] for detail arguments). Thus $\left|F^{(2) \lambda}\right|$ has subdominant energy dependence: $(\ln s)^{\lambda-1}$ ( compared to $\left.\left|F^{(1) \lambda}\right| \sim(\ln s)^{\lambda}\right)$. Therefore,

$$
\left|F^{\lambda}(s, t)\right| \leq A_{2}\left(\frac{1}{|t|}\right)^{\frac{1+\lambda}{2}}\left(\frac{1}{T_{0}}\right)^{\lambda / 2} s^{1+(N-1) \sqrt{\frac{|t|}{T_{0}}}}(\ln s)^{\lambda}
$$

Remarks: (R1.1) The bound holds in the domain $|t|<T_{0}$, including a part of the physical region $t<0$. (R1.2) This is generalization of the bound for the $D=4$; note the power dependence and on $|t|$. The known result [5] is recovered, setting $\lambda=1 / 2$. (R1.3) An important and noteworthy point is the appearance of Regge-like power law in (20), in the domain $0<|t|<T_{0}$ and the power of $s$ is $\lambda$-independent. It is a reminiscence of open string tree amplitude in the Regge limit. (R1.4) The bound is a substantial improvement over that of $[10,11]$ since theirs was only for $\left|F^{\lambda}(s, 0)\right|$. A bound on number of zeros of $F^{\lambda}(s, t)$ follows from $(20)$. 
Lemma 2. The number, $n_{r}(s)$, of zeros of $F^{\lambda}(s, t)$ within the disk $|t|<r<T_{0}$ is bounded from above by

$$
n_{r}(s) \leq \frac{e \sqrt{r}}{2 \sqrt{T_{0}}} \ln s
$$

The terms of $o\left((l n s)^{-1}\right)$ in the r.h.s of $(21)$ are ignored.

Proof. According to Jensen's theorem [17], $n_{r}(s)$ satisfies the inequality

$$
n_{r}(s) \leq \frac{1}{\ln 1 / \delta} \operatorname{Max} \ln \left|\frac{F^{\lambda}(s, r / \delta)}{F^{\lambda}(s, 0)}\right|, \quad r / T_{0}<\delta<1
$$

Note that $\left|F^{\lambda}(s, 0)\right|=\sqrt{\left(\operatorname{Re} F^{\lambda}(s, 0)\right)^{2}+\left(\operatorname{Im} F^{\lambda}(s, 0)\right)^{2}}$ and $\left|F^{\lambda}(s, 0)\right| \geq s \sigma_{t}$ as per our normalization: $\operatorname{Im} F^{\lambda}(s, 0)=s \sigma_{t}$. Therefore,

$$
n_{r}(s) \leq \frac{1}{\ln 1 / \delta} \operatorname{Max}\left(F^{\lambda}\left(s, \frac{r}{\delta}\right) \frac{1}{s \sigma_{t}}\right)
$$

by the virtue of (20), on the r.h.s we are left with

$$
\ln \left[C_{2} \exp \left(\left\{(N-1) \sqrt{\frac{r / \delta}{T_{0}}} \ln s\right\}\right)\left(\frac{1}{r / \delta}\right)^{\frac{1+\lambda}{2}}\left(\frac{1}{T_{0}}\right)^{\lambda / 2} \frac{1}{\sigma_{t}}\right]
$$

Retaining the leading order term in $\operatorname{lns}$

$$
\begin{aligned}
& \ln \left|\frac{F^{\lambda}(s, r / \delta)}{F^{\lambda}(s, o)}\right| \leq \ln s\left[(N-1) \sqrt{\frac{r}{\delta}}+\frac{1}{(\ln s)}\left\{\ln C_{1}+\right.\right. \\
&\left.\left.\lambda \ln \ln s-\left(\frac{(\lambda+1)}{2}\right) \frac{r}{\delta}-\frac{\lambda}{2} T_{0}-\ln \left(\sigma_{t}\right)\right\}\right]
\end{aligned}
$$

No rigorous lower bound exists on $\sigma_{t}$ for $D>4$ like the Jin-Martin [18] bound: $\sigma_{t} \geq$ $s^{-6}$ in $D=4$. Even if it existed, its contribution to (25) will be $(\ln s)^{-1} \ln \sigma_{t} \sim o(1)$. Thus retaining the lns term, in large $s$ limit, is justified in estimation of the upper bound on $\ln \left|F^{\lambda}(s, r / \delta) / F^{\lambda}(s, 0)\right|$; consequently,

$$
n_{r}(s) \leq \frac{(N-1) \sqrt{\frac{r}{\delta}}}{(\ln 1 / \delta) \sqrt{T_{0}}} \ln s
$$

follows from (22). Optimizing (25) with respect to $\delta$ leads to the desired bound (21). Corollary: There are no zeros inside a disk of radius $r_{0}$ in the $t$-plane and

$$
r_{0}<\frac{C_{2}}{(\ln s)^{2}}
$$

Remarks: (R2.1) $C_{2}=T_{0}[e(N-1)]^{-2}$, is independent of $s$. The correction to (25) is order $o(1)$ if Jin-Martin exists for $D>4$. (R2.2) There is a zero-free disk with 
shrinking radius. More importantly, to leading order in $l n s$, the upper bound on $r_{0}$ is $D$-independent. (R2.3) There is a single zero in the $|t|$-plane in an annular region:

$$
r_{0}(s)<\frac{C_{2}}{(\ln s)^{2}}<r_{1}(s)<\frac{C_{3}}{(\ln s)^{2}}
$$

$C_{3}$ being another s-independent constant. We recall the study of the zeros of amplitudes has played a crucial role in deriving many rigorous results in the past $[19,20,21]$. The bound on $A^{\lambda}(s, t)=\operatorname{Im} F^{\lambda}(s, t)$ can be improved substantially in the unphysical region $0<t \leq T_{0}$, utilizing unitarity, properties of Gegenbauer polynomials and the bound (20). The important point to note is that $A^{\lambda}(s, t)>0$ for $t>0$ as evident from (6) since $0 \leq \operatorname{Im~}_{l}^{\lambda} \leq 1$ and $C_{l}^{\lambda}(x)>0$ for $x>1$. We arrive at the final result through following steps. The zeros of Jacobi polynomial $P_{l}^{(\alpha, \beta)}(\cos \theta)$, for $\alpha>-1, \beta>-1$ satisfy following properties: ( Theorem 8.9.1 of [13]), let $0<\theta_{1}<\theta_{2}, \ldots,<\theta_{l}<\pi$ be zeros of $P_{l}^{(\alpha, \beta)}(\cos \theta)$ (it applies to $C_{l}^{\lambda}(\cos \theta)$ since $\alpha=\beta=\lambda>1 / 2$ ). Then

$$
\theta_{\nu}=\frac{1}{l}(\nu \pi+O(1))
$$

with $O(1)$ being uniformly bounded constant for all values of $\nu=1,2, \ldots, l ; l=$ $1,2, \ldots$ Thus two consecutive zeros are separated [13] as $\theta_{\nu+1}-\theta_{\nu}=\frac{\pi}{l}$. The distribution of zeros of Legendre polynomial (a special case of Gegenbauer polynomial with $\lambda=1 / 2$ ) was crucially used by Kinoshita to derive an important bound [22] on absorptive amplitude, for $D=4$, in the region $0<t<t_{0}$. We recall that the Gegenbauer polynomials are the ultraspherical Jacobi polynomials and the distribution of the zeros of the Gegebauer polynomials are governed by the theorem stated above. Therefore, for the case at hand, the spacing of zeros (28) is also quite analogous. Factorize, $C_{l}^{\lambda}(z)=\Pi_{\nu=1}^{l}\left[\frac{\left(z-z_{\nu}\right)}{1-z_{\nu}}\right], z_{\nu}=\cos \theta_{\nu}$ are the location of the zeros. $A^{\lambda}(s, t)$ becomes a complex function when $t$ is complex i.e. when $C_{l}^{\lambda}(z)$ becomes complex. Thus if $\boldsymbol{\Phi}$ is the phase of $C_{l}^{\lambda}(z)$ for $z=1+a+i b$, we look for the curve in $z$-plane which intersects the ellipse where $C_{l}^{\lambda}(z)$ hits its first zero i.e. $\boldsymbol{\Phi}=\pi / 2$. For complex $t$, decompose $t=u+i v, u$ and $v$ being the real and imaginary parts of $t$. There is a small domain, $\mathcal{D}$, in the upper half $t$ plane, which is intersection of this curve with the ellipse such that $\operatorname{Re} A^{\lambda}(s, t)$ is positive inside $\mathcal{D}$. Recall that as we go off real $t$ axis into this domain $A^{\lambda}(s, t)$ becomes complex. The arguments of [22] can be adopted to show that $\mathcal{D}$ is given by

$$
|v| \leq \frac{\pi \sqrt{u}}{2 C_{4} \ln s}
$$

$C_{4}$ is $s$-independent constant. Thus $\operatorname{Re} A^{\lambda}(s, t)$ is a positive, harmonic function inside $\mathcal{D}$. Consequently, a powerful theorem on positive harmonic functions holds [23]. Now define $R_{0}, 0<R_{0}<T_{0}-\delta$, such that it is within $\mathcal{D}$. Consider a disk 
$\mid t-R_{0}<\pi\left(\sqrt{R_{0}}\right) /\left(2 C_{4}\right.$ lns $)$ which is shrinking as lns, note that $t>0$.

The Harnack's theorem [23] states: For any $t$ in the smaller disk

$$
\left|t-R_{0}\right|<\frac{\pi r \sqrt{R_{0}}}{2 C_{4} \ln s}, \quad 0<r<1
$$

with $r$ inside the disk, the positive, harmonic function, $\operatorname{Re} A^{\lambda}(s, t)$ is bounded from above and below as

$$
\frac{1-r}{1+r} A^{\lambda}\left(s, R_{0}\right)<\operatorname{Re} A^{\lambda}(s, t)<\frac{1+r}{1-r} A^{\lambda}\left(s, R_{0}\right)
$$

$A^{\lambda}\left(s, R_{0}\right)$ is real and positive as argued before. $A^{\lambda}(s, t)$ is defined inside the circle which lies in $\mathcal{D}$ and hence $\operatorname{Re} A^{\lambda}(s, t)>0$. Note that the radius is order $\frac{1}{\ln s}$, therefore, inequality (31) conveys that $\operatorname{Re} A^{\lambda}(s, t)$ will not increase by more than a finite factor when $t$ is increased by $\frac{1}{\ln s}$. We can derive various bounds on $A^{\lambda}(s, t)$ and its derivatives through applications of theorem (31) inside the disk (30).

An upper bound on $b(s)$ is derived utilizing the bound (20) and the inequality $\left|F^{\lambda}(s, 0)\right| \geq s \sigma_{t}$. It follows from Cauchy's inequality and suitable adaptation of the arguments of $[21,24]$. If the zero-free radius shrinks less rapidly than (26) i.e. $r_{0}(s)>(\ln s)^{-2+\eta}, \eta>0$, then bounds on $b(s)$ and $\sigma_{t}$ are improved [24].

We conclude this note with following remarks and observations in the context of higher dimensional theories. There are scenarios where higher dimensional theories admit a low compactification scale, $\sqrt{\hat{s}} \sim$ a few $\mathrm{TeV}$. The phenomenological consequences of such models have been explored extensively [26, 27]. Petrov [29] has argued that the cross section of a $D>4$ theory will have same behavior as that of a $D=4$ theory below the compactification scale. He supported this claim through model calculations. We mention in passing that the S-matrix has interesting analyticity properties in the energy plane in case of potential scattering when some spatial dimensions are compactified [30,31]. These issues have not been thoroughly investigated in field theoretic frame work.

Let us consider a higher dimensional theory with a low energy scale of compactification, $\hat{s}$. When the theory is probed with energies below the compactification scale it will behave like a $D=4$ theory as argued by Petrov. However, above the scale $\hat{s}, \sigma_{t}$ of a $D>4$ theory might behave as if (4) is violated, although $\sigma_{t}$ does not necessarily have to violate $D=4$ bound (4). Thus it might be worth while to explore qualitatively whether $\sigma_{t}$ admits an $s$-dependence: $\left(\ln \frac{s}{\hat{s}}\right)^{\beta}, \beta>2$ at the extremely high energies accessed by LHC and in cosmic ray experiements. If the total cross section data for extreme high energies show such an energy dependence then one might get a hint of decompactification at lower scale as alluded to earlier. Moreover, there are other avenues to test unitarity bounds on quantities listed in (5) for $D=4$. They will be measured with accuracy at LHC from $s=36 \mathrm{TeV}^{2}$ to $s=196 \mathrm{TeV}^{2}$ at LHC. Moreover, $b(s)$ and $\mathbf{h}_{d}$ are bounded by $(\ln s)^{2}$; as $\mathbf{h}_{d}$ grows with energy, the width of $\frac{d \sigma}{d t}$ shrinks $[5,21]$. Thus precision measurements of $b(s), \mathbf{h}_{d}$ and other measurable 
quantities listed in (5) will stringently test unitarity constraints at LHC. An unambiguous deviation from unitarity bounds, derived for $D=4$ theories, might provide an indirect evidence for existence of higher dimensional theories.

Acknowledgments: I am grateful to I. Antoniadis for valuable suggestions. I thank S. B. Giddings and R. Sasaki for useful correspondence. I am thankful to Sanjay Swain for suggestions and for his critical remarks. The warm hospitality Hermann Nicolai and the Albert Einstein Institute is gratefully acknowledged. 


\section{References}

1. W. Heisenberg, Zeitschrift für Physik, 13365 (1952)

2. A. Martin, Scattering Theory: unitarity, analyticity and crossing, SpringerVerlag, Berlin-Heidelberg-New York, (1969).

3. A. Martin and F. Cheung, Analyticity properties and bounds of the scattering amplitudes, Gordon and Breach, New York (1970).

4. R. J. Eden, Rev. Mod. Phys. 43, 15 (1971)

5. S. M. Roy, Phys. Rep. C5, 125 (1972).

6. H. Epstein, V. Glaser and A. Martin, Commun. Math. Phys. 13, 275 (1969).

7. M. Froissart, Phys. Rev. 123, 1053 (1961)

8. A. Martin, Nuov. Cimen. A42, 930 (1966)

9. A. Martin and S. M. Roy, Phys. Rev. D89, 045015 (2014); A. Martin and S.

M. Roy, Phys. Rev. D91, 076006 (2015).

10. M. Chaichian and J. Fischer, Nucl. Phys B303, 557 (1988).

11. M. Chaichian, J. Fischer and Yu. S. Vernov, Nucl. Phys. bf B383, 151 (1992).

12. M. Soldate, Phys. Lett. B197, 321 (1987).

13. G. Szego, Orthogonal Polynomials, American Mathematical Society, New York,1959.

14. H. Bateman and A. Erdelyi, Higher Trascedental Functions, Vol I, McGraw Hill, New York, (1953).

15. H. Lehmann, Nuov. Cimen. 10, 579 (1958).

16. A. Martin, Nuov. Cimen. 42, 930 (1966)

17. E. C. Titchmarsh, The theory of functions, Oxford University Press, London (1939), p171.

18. Y. S. Jin and A. Martin, Phys. Rev. 135, B1369 (1964).

19. J. D. Bessis, Nuov. Cimen. 45A, 974 (1966).

20. G. Auberson, T. Kinoshita and A. Martin, Phys. Rev, D3, 3185 (1971).

21. R. J. Eden and G. D. Kaiser, Nucl. Phys. bf B28, 253 (1971). 
22. T. Kinoshita, Phys. Rev. 152, 1266 (1966)

23. C. Carathéodory, Theory of Functions (Chelsea Publishing Company, New York, 1964), p.153.

24. J. Maharana, Commun. Math. Phys. 58, 195 (1978)

25. T. Kinoshita and J. Maharana, J. Math. Phys. 16, 2294 (1975).

26. I. Antoniadis, Phys. Lett. B246, 377 (1990).

27. I. Antoniadis, N. Arkani-Hamed, S. Dimopoulos and G. Dvali, Phys. Lett. 436, 257 (1998). For comprehensive list of references see L. A. Anchordoqui, I. Antoniadis, W-Z Feng, H. Goldberg, X. Huang, D. Lust, D. Stojkovich, and T. R. Taylor, Phys. Rev. D90, 066013 (2014).

28. I. Antoniadis, private communication.

29. V. A. Petrov, Mod. Phys. Lett. A16, 151 (2001).

30. N. N. Khuri and T. T. Wu, Phys. Rev. D56, 6779 (1997)

31. A. Martin, Commun. Math. Phys. 219, 191 (2001). 